\title{
HOMOGENIZATION OF FIBER REINFORCED ELASTOMER LAMINATES
}

\author{
Tilen Ceglar $^{1}$ and Heinz E. Pettermann ${ }^{1 *}$ \\ ${ }^{1}$ Institute of Lightweight Design and Structural Biomechanics, TU Wien, Vienna, Austria \\ * pettermann@ilsb.tuwien.ac.at
}

The effective hyperelastic anisotropic response of a cross-ply laminate built by unidirectional fiber reinforced elastomer plies is studied. The matrix is described by Ogden type hyperelasticity and the fibers are taken to be linear elastic with a volume fraction of $40 \%$. Two homogenization strategies are employed in the context of the Finite Element Method using the software package Abaqus/Standard 2020 (Dassault Systèmes Simulia Corp., Providence, RI, USA).

First, a plane periodic unit cell of the $[90 / 0]_{\mathrm{S}}$ laminate is set up whereby the matrix-fiber microstructure is resolved over the entire thickness, Fig. 1. For the second approach, the laminate unit cell is simplified with respect to the ply representation. Here, the effective behavior of the plies is modeled by the anisotropic hyperelastic Holzapfel-Gasser-Ogden constitutive law available in Abaqus. Its parameters can be calibrated by recourse to homogenization of small size unit cells or by fitting to experimental data.

A number of in-plane load cases are studies and the non-linear responses of the different modeling schemes are compared. Good agreement is found for moderate strains up to some $40 \%$. Consequently, the simpler model can serve in large scale structural analyses without compromising the quality of the results.

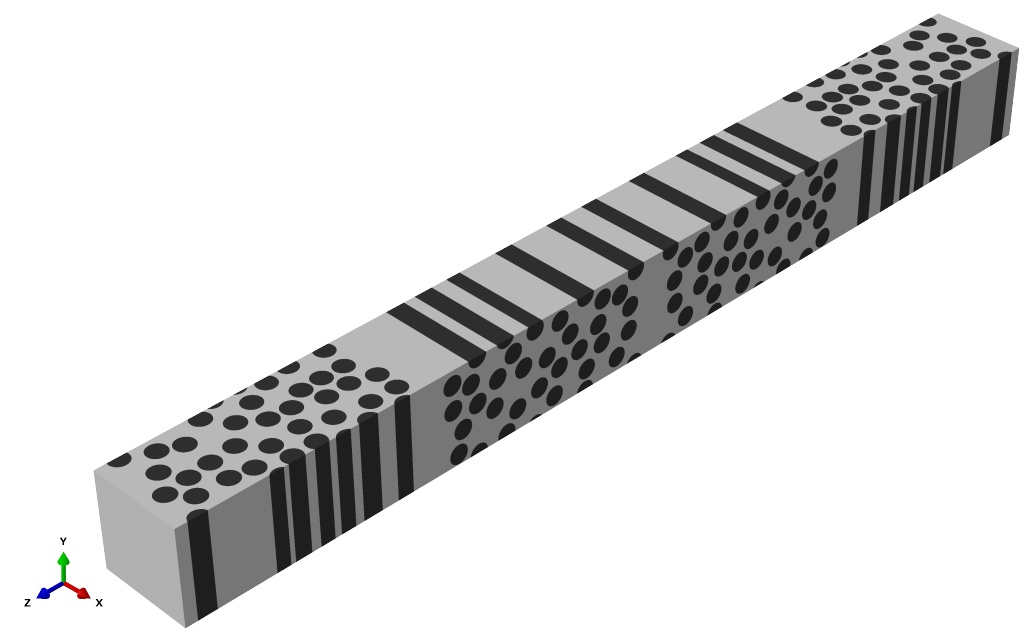

Figure 1. Plane periodic unit cell representing the entire thickness of the laminate.

\section{Acknowledgements}

The funding by the Polymer Competence Center Leoben GmbH (PCCL) within the COMETprogram by the Austrian Federal Ministry for Transport, Innovation, and Technology, the Austrian Federal Ministry of Digital and Economic Affairs, Österreichische Forschungsförderungsgesellschaft mbH (FFG), the Provinces of Styria, Lower Austria, and Upper Austria, is gratefully acknowledged. 